\title{
Elements of nanocrystal high-field carrier transport modeling
}

\author{
C. Sevik* and C. Bulutay ${ }^{1}$ \\ Department of Physics, Bilkent University, 06800 Ankara, Turkey \\ Received 30 July 2006, revised 14 August 2006, accepted 14 August 2006 \\ Published online 7 February 2007
}

PACS 61.46.Hk, 72.20.Ht, 73.63.Bd

Embedded semiconductor nanocrystals (NCs) within wide bandgap oxide materials are being considered for light emission and solar cell applications. One of the fundamental issues is the high-field transport in NCs. This requires the combination of a number of tools: ensemble Monte Carlo carrier transport simulation, $a b$ initio band structure of the bulk oxide, Fermi's golden rule modeling of impact ionization and Auger processes and the pseudopotential-based atomistic description of the confined NC states. These elements are outlined in this brief report.

(C) 2007 WILEY-VCH Verlag GmbH \& Co. KGaA, Weinheim

Silicon nanocrystals (NCs) have become a major research area due to all-silicon photonics prospects [1]. A relatively less explored subject is the high-field transport phenomena in Si NCs embedded in oxide matrix. This research has direct implications for the electroluminescence and carrier multiplication as a means of increase of efficiency in NC-based solar cells. In this work we present the elements of the high-field transport modeling in NCs.

Our general framework of high-field transport modeling in NCs is summarized in Fig. 1. Carriers can either be injected from the contacts to delocalized bulk states of the oxide matrix via Fowler-Nordheim tunneling (FNT) or can directly tunnel (DT) to NC confined states depending on the barrier thickness (cf. Fig. 1(a)). Those carriers within the confined NC states can resonantly tunnel (RT) to neighboring NCs which can also be accompanied by phonon absorption/emission; sometimes these channels are generally termed as hopping transport (HT). This in general creates two tracks of current: via NCs in the form of tunneling and also over the bulk oxide states as depicted in Fig. 1(b). The carriers in these two tracks can be interchanged mainly by the two quantum processes: impact ionization (II) and Auger processes. To discriminate the II process in bulk oxide states from those involving the NC electrons the latter is sometimes named as quantum-confined II (QCII). These elements will be discussed next.

For the high-field transport phenomena the ensemble Monte Carlo (EMC) technique is currently the most reliable choice, free from major simplifications. We include the following scattering mechanisms in our EMC treatment: acoustic and optical deformation potential, polar optical phonon, ionized impurity and the impact ionization scatterings; furthermore, the Pauli exclusion principle needs to be enforced as well $[2,3]$. On the other hand, the piezoacoustic, neutral impurity and dislocation scatterings are not included as they become significant at low temperatures and fields [4]. We simulate the hot-carrier transport of both electrons and holes within $\mathrm{SiO}_{2}$ up to an applied field of $12 \mathrm{MV} / \mathrm{cm}$. As shown in Fig. 2 the energy gained by the holes is well below $0.5 \mathrm{eV}$ even for fields above $10 \mathrm{MV} / \mathrm{cm}$ due to excessive scattering which is a consequence of the very large density of states (DOS) close to the valence band edge.

The hot carriers gain substantial energy under high applied field and increase their energies by several electronvolts. For this reason, preferably the full band structure or at least the full DOS information needs to be supplied to the EMC simulation. Figure 2 (b) displays the first-principles band structure for the $\alpha$ quartz phase of $\mathrm{SiO}_{2}$ which is computed within the density functional theory, using the pseudopotential

* Corresponding author: e-mail: sevik@fen.bilkent.edu.tr, Phone: +9031229021 44, Fax: +90 3122664579 


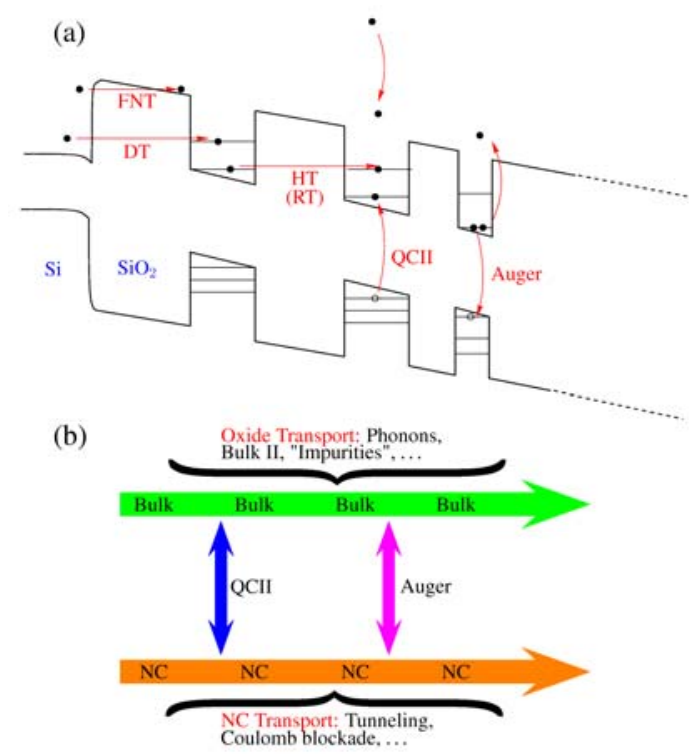

Fig. 1 Schematic presentation of major high-field quantum processes in embedded NCs.
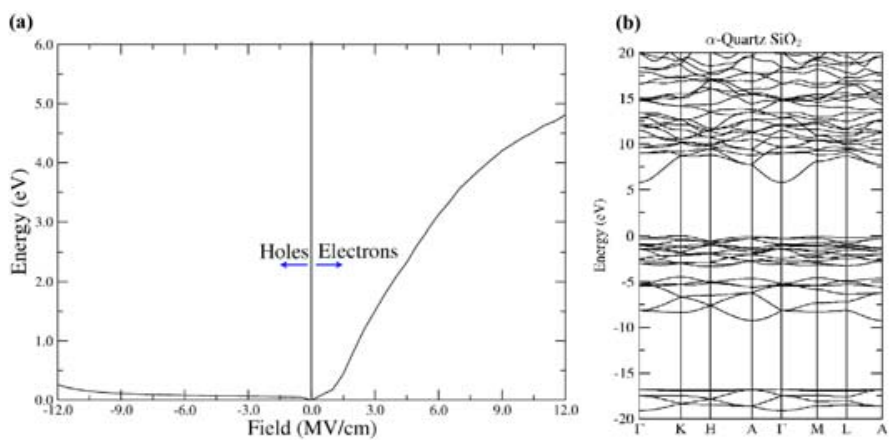

Fig. 2 a) EMC results of field vs energy profile for electrons and holes in $\mathrm{SiO}_{2}$, b) ab initio band structure for $\alpha$-quartz phase of $\mathrm{SiO}_{2}$.

method employing the local density approximation [5]. The contrast between the DOS of conduction and valence bands can be observed.

Two important processes that connect the carriers within the bulk oxide states and the confined NC states are the II and Auger processes. Both of them are essentially screened Coulomb scattering events; in the case of Auger, there occurs a non-radiative NC electron-hole recombination with the energy given to a third carrier whereas in II, a NC electron-hole pair is generated through an 'impacting' bulk oxide hot-carrier (cf. Fig. 1). The rates of these process can be obtained by the Fermi's golden rule:

$$
P\left(E_{1}\right)=\frac{2 \pi}{\hbar} \sum_{\mathrm{NCs}} \sum_{2,1^{\prime}, 2^{\prime}}\left|M_{12 \rightarrow 1^{\prime} 2^{\prime}}\right|^{2} \delta\left(E_{1}-E_{1^{\prime}}+E_{2}-E_{2^{\prime}}\right)
$$

where,

$$
M_{12 \rightarrow 1^{\prime} 2^{\prime}}=\int d^{3} \mathbf{r}_{1} d^{3} \mathbf{r}_{\mathbf{2}} \psi_{1^{\prime}}^{*}\left(\mathbf{r}_{1}\right) \psi_{2^{\prime}}^{*}\left(\mathbf{r}_{2}\right) V_{\mathrm{scr}}\left(\left|\mathbf{r}_{1}-\mathbf{r}_{\mathbf{2}}\right|\right) \psi_{1}\left(\mathbf{r}_{1}\right) \psi_{2}\left(\mathbf{r}_{2}\right),
$$


is the matrix element. Here $E_{1}$ and $E_{1^{\prime}}$ are the initial and final states of carrier-1, and $E_{2}$ and $E_{2^{\prime}}$ are those of carrier-2. Also, the dielectric screening of the Coulomb interaction $V_{\text {scr }}$ between the 'colliding' carriers 1 and 2 deserves attention as the embedded NC dielectric permittivity is bulkline inside the NC and is effectively replaced by the host dielectric constant of outside medium; we refer to Ref. [6] and references therein for essential details. Finally, in these expressions $\psi_{i}$ 's are the wave functions of the involved carriers. For the bulk oxide states well above the mobility edge they are of the well-known Bloch form. However, the NC states need more elaboration as described next.

The II and Auger are typical examples for demonstrating the analysis of quantum processes which finally end up with the computation of certain transition matrix elements, hence requires the knowledge of wave functions. Accurate description of $\mathrm{NC}$ wave functions is properly done with an atomistic Hamiltonian. The empirical pseudopotential-based approach proposed by Wang and Zunger [7] is particularly suitable for our purposes [8]. The technique is based on the expression of the NC states as the linear combination of the bulk bands of the constituent semiconductors. Due to lack of space we refer to Refs. [7, 8] for details. In Fig. 3 we display the wave functions of the highest occupied molecular orbital (HOMO) and the lowest unoccupied molecular orbital (LUMO) for an embedded $2.7 \mathrm{~nm} \mathrm{Si} \mathrm{NC}$. The oscillations within the envelope reflect the underlying atomistic structure.

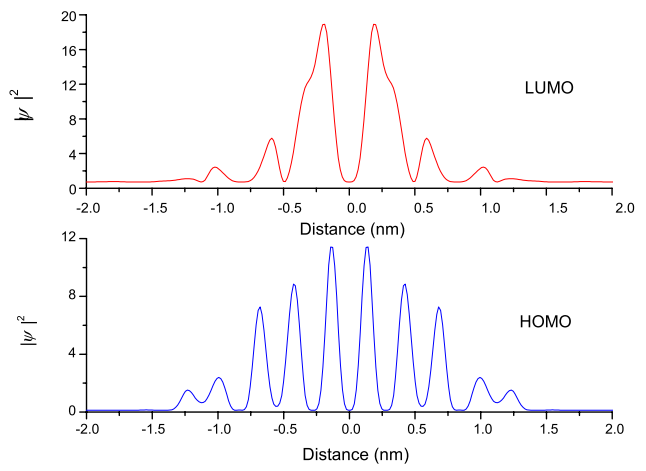

Fig. 3 Pseudopotential wave functions for the HOMO and LUMO states of a $2.7 \mathrm{~nm}$-diameter Si NC.

The subject of carrier dynamics under high field requires rigorous treatment without simplifying assumptions which is what we aim in our work in progress. In this brief report we have outlined the tools for high-field transport modeling of embedded NCs which can act as a guideline for future research.

Acknowledgements This work has been supported by the European FP6 Project SEMINANO with the contract number NMP4 CT2004 505285 and by the Turkish Scientific and Technical Council TÜBİTAK with the project number $106 \mathrm{~T} 048$.

\section{References}

[1] L. Pavesi and D. J. Lockwood (Eds.), Silicon Photonics, Topics in Applied Physics, No. 94 (Springer-Verlag, Berlin, 2004).

[2] C. Sevik and C. Bulutay, Appl. Phys. Lett. 83, 1382 (2003).

[3] C. Bulutay, B. K. Ridley, and N. A. Zakhleniuk, Phys. Rev. B 68, 115205 (2003).

[4] B. K. Ridley, Quantum Processes in Semiconductors, 4th ed. (Oxford University Press, Oxford, 1999).

[5] C. Sevik and C. Bulutay, submitted for publication (2006).

[6] X. Cartoixà and L. -W. Wang, Phys. Rev. Lett. 94, 236804 (2005).

[7] L. -W. Wang and A. Zunger, Phys. Rev. B 59, 15806 (1999).

[8] C. Bulutay, J. Nanosci. Nanotechnol., accepted for publication (2006). 